\title{
Correlation of Successive Atomic Steps in Crystals by Relaxation Mode Analysis
}

\author{
A. D. Franklin*
}

(March 5, 1965)

\begin{abstract}
The relaxation mode analysis is used to compute the average value of the cosine of the angle between successive steps of an ion diffusing by a vacancy mechanism on a lattice. The technique is applicable to tracers (self-diffusion) or impurities, on any lattice. The result, given in terms of the eigenvalues and eigenvectors of a secular equation, is suitable for machine computation. Sample computations for self-diffusion on an fec lattice illustrate the method. It is necessary, in order to do the calculations, to have a lattice of finite size, the boundary of which is chosen either totally reflecting or completely transparent to vacancies. For self-diffusion, the boundary can be centered on the vacancy, and convergence of the computed value with boundary radius is rapid. For impurity diffusion, the boundary must be centered on the impurity, and convergence is much slower.
\end{abstract}

\section{Introduction}

Diffusion of atoms or ions through a crystal by the vacancy or interstitialcy mechanisms proceeds not by random-walk steps but by steps that are to some extent correlated with each other $[1] .^{1} \quad$ The degree of correlation between successive steps can be represented by $\overline{\cos \theta_{\alpha}}$, where $\theta_{\alpha}$ is the angle between the paths of the $\alpha$ th step and the $(\alpha+1)$ th step. If the term "tracer" is defined to mean any moving particle in the crystal, then where the tracer is a host ion moving upon its own sublattice, as in self-diffusion, $\overline{\cos \theta_{\alpha}}$ depends only upon the geometry of the lattice. If the tracer is an impurity, $\overline{\cos \theta_{\alpha}}$ depends as well upon the way in which the impurity influences the motion of the vacancy in its vicinity.

A number of methods have been developed for computing $\overline{\cos \theta_{\alpha}}[2-6]$. As shown by Compaan and Haven [3], the computations are very much simplified if use is made of the crystal symmetry. It is the purpose of this paper to show how maximum use can be made of the symmetry by employing the technique of relaxation mode analysis [7-10] to calculate $\overline{\cos \theta_{\alpha}}$. The method given is perfectly general, applying to both self-diffusion and impurity diffusion in any crystal lattice by the vacancy mechanism. With minor modification it could be applied to the interstitialcy mechanism as well. It is cast in a form that is extremely convenient for machine computation.

*Work done while on a training assignment to the Theoretical Physies Division, UKAERE, Harwell, England. Permanent address: National Bureau of Standards, Washington, D.C.

1 Figures in brackets indicate the literature references at the end of this paper.

\section{Theory}

The development of the method will be given for any lattice, but the fec lattice will be used to illustrate the development and the method itself. The problem can be visualized with the aid of figure 1 . This shows the tracer at the center of a cube, with the nearest neighbor sites in the fcc lattice in the middle

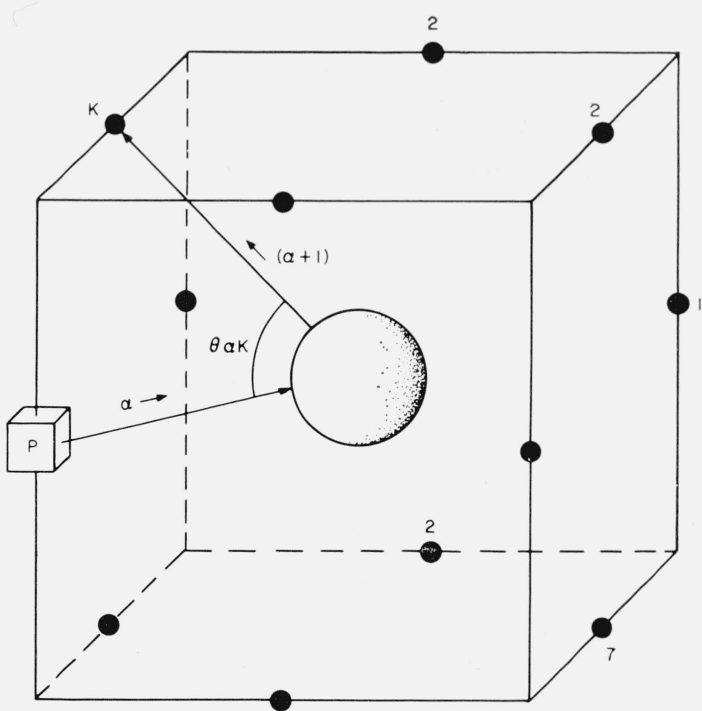

Figure 1. Successive tracer steps in the fcc lattice.

The tracer is at the center, after the $\alpha$ th step which has left the vacancy at $P$. A possible $(\alpha+1)$ step is shown, to one of the twelve nearest neighbor sites, making an angle $\theta_{\alpha k}$ with the $\alpha$ th step. For self-diffusion, only two kinds of nearest neighbor sites, here marked 1 and 2, need be considered. (Eq 12.) 
of the edges of the cube. The tracer has just completed the step shown by the arrow labeled $\alpha$, leaving the vacancy in the position labeled $p$. In addition, one of the twelve possibilities for the $(\alpha+1)$ th step is shown. After the $\alpha$ th step, the vacancy will move in some fashion. It may immediately reexchange with the tracer, or it may first move directly or indirectly to one of the eleven other positions next to the tracer and then reexchange with the tracer. Or it may move away altogether and not return, so that the next step of the tracer is an exchange with a different vacancy. A different vacancy will approach the tracer in a random manner, provided the original vacancy is far away when the new one approaches, so that successive steps in which different vacancies figure are not correlated. Only steps involving the same tracer need be considered in calculating $\overline{\cos \theta_{\alpha}}$ for low vacancy concentrations. The problem is solved when the probability of each one of the twelve candidates for the $(\alpha+1)$ th step is calculated. Although figure 1 shows only one set of lattice sites from which the vacancy can make a direct exchange with the tracer, in general there may be several, the sites in each set being crystallographically equivalent in the presence of the tracer. The solution of the problem can be found either by considering the average behavior of a large number of identical systems, or by thinking in terms of the probability of finding the vacancy on a given site for one system, as done by Bardeen and Herring [1] and others. This latter view will be taken here.

Let the probability that the vacancy occupies the $k$ th site at a time $t$ after the $\alpha$ th step be $P_{k}(t)$. Also, let $w_{k 0}$ be the frequency with which a vacancy occupying the $k$ th site will change places with the tracer at the origin. Then

$$
\overline{\cos \theta_{\alpha}}=\sum_{k} w_{k 0} \cos \theta_{\alpha k} \int_{0}^{\infty} P_{k}(t) d t .
$$

In the relaxation mode analysis, eq (1a) can be written as a vector equation, involving vectors in site-space, to be defined below. One vector contains all of the time-dependence of the probabilities $P_{k}(t)$, the other the geometrical quantities $\cos \theta_{\alpha k}$. When the basis for these vectors is properly chosen, the time dependence becomes a simple series of exponential decay functions, for which the integrations are readily performed, and eq (1a) reduces to a rather simple form.

Site space may be defined by considering each site to correspond to a vector in a basis set that spans the space. Any set of scalars assigned to the sites can be represented by a vector in this space, with the scalars for coordinates. If there are $N$ accessible lattice sites, then the site space is $N$-dimensional. Equation (1a) may then be written rather compactly in terms of two vectors

$$
\overline{\cos \theta_{\alpha}}=\int_{0}^{\infty} \boldsymbol{\tau}^{\prime} \cdot \mathbf{P}(t) d t,
$$

where the coordinates of $\boldsymbol{\tau}^{\prime}$ are $w_{k 0} \cos \theta_{\alpha k}$ and the coordinates of $\mathbf{P}(t)$ are the time-dependent probabilities of finding the vacancy on the various sites.

The process of identifying the proper basis can be started by dividing the lattice sites into shells, the sites in each shell being equivalent to each other under the site-group, which is the group of rotational (proper and improper) symmetry operations of the crystal about the tracer as origin. Each shell defines a subspace of the whole site-space, each subspace being orthogonal to all the subspaces defined by the other shells. A vacancy on any site in one of the first few shells, counting outward from the origin, can make a direct exchange with the tracer. For a vacancy on a site in the $n$th such shell, the frequency $w_{n 0}$ with which the vacancy would change places with the tracer is the same for all sites. Hence $\tau^{\prime}$ can be written

$$
\boldsymbol{\tau}^{\prime}=\sum_{n} w_{n 0} \tau_{n}^{\prime}
$$

where the summation is over all shells from which direct exchange of the vacancy with the tracer can occur, and $\boldsymbol{\tau}_{n}^{\prime}$ is the vector whose coordinates are $\cos \theta_{\alpha k}$ for all sites in the $n$th shell and zero for all others. Then eq $(1 \mathrm{~b})$ becomes

$$
\overline{\cos \theta_{\alpha}}=\sum_{n} w_{n 0} \int_{0}^{\infty} \boldsymbol{\tau}_{n}^{\prime} \cdot \mathbf{P}(t) d t .
$$

The vector $\mathbf{P}(\mathrm{t})$. Since all of the time-dependence is contained in the vector $\mathbf{P}(t)$, only this need be considered in identifying the desired basis. The components of $\mathbf{P}(t)$, which are the probabilities $P_{k}(t)$, are governed by the set of $N$ rate equations

$$
\frac{d P_{k}}{d t}=-\sum_{l}^{N}\left(w_{k l} P_{k}-w_{l k} P_{l}\right)
$$

where $w_{k l}$ is the frequency with which a vacancy on the $k$ th site will make a single, direct move to the $l$ th site. In this problem, the tracer at the origin is treated as a sink for probability - the probability of a vacancy moving to the origin from an adjacent site is positive, but the probability of the return step is taken as zero. Equation (2) can be written in terms of the vector $\mathbf{P}(t)$ in site space:

$$
\frac{d \mathbf{P}}{d t}=C \mathbf{P}(t)
$$

where $C$ is the matrix of the $w_{k l}$ in eq (2). The solutions to eq (3) are

$$
\mathbf{P}(t)=\sum_{q} \sum_{s} f_{q s} \mathbf{u}_{q s} \exp \left(-\lambda_{q s} t\right)
$$

where the $f_{q s}$ are defined by the initial distribution of probability, at $t=0$, the $\mathbf{u}_{q s}$ are the eigenvectors of the matrix $C$, and the $\lambda_{q s}$ are the corresponding eigenvalues, which are also the inverse relaxation times. The subscripts $q$ and $s$ are defined below.

Use is made of the irreducible representations of the site-group in finding the eigenvectors and eigen- 
values. The subscript $s$ denotes the various irreducible representations, some of which may be of dimension greater than one. For these, several values of $s$, one for each dimension, will be required. The subscript $q$ denotes one of the several eigenvectors (and eigenvalues) belonging to one dimension of a given irreducible representation. To each dimension of an irreducible representation of the sitegroup, there corresponds a subspace of the whole site-space, spanned by a set of basis vectors $\mathbf{V}_{m s}$. Each eigenvector $\mathbf{u}_{q s}$ can be written as a linear combination of the basis vectors in one set:

$$
\mathbf{u}_{q s}=\sum_{m} a_{m q s} \mathbf{V}_{m s}
$$

Any basis vector $\mathbf{V}_{m_{s}}$ lies wholly within the subspace defined by one shell of sites, although several basis vectors in a set may be drawn from the subspace of the same shell. In this case, the shell has been divided into subshells by the irreducible representation, and the indices $n$ in eq (1c) and $m$ in eq (5) specify the subshell.

The constants $f_{q s}$, etc., can be found from the initial position $x_{0}, y_{0}, z_{0}$ of the vacancy. At $t=0$

$$
\mathbf{P}(0)=\sum_{q} \sum_{s} f_{q s} \mathbf{u}_{q s}
$$

so that, since the eigenvectors are all mutually orthogonal, and using eq (5),

$$
\mathbf{f}_{q s}=\mathbf{P}(0) \cdot \mathbf{u}_{q s}=\sum_{m} a_{m q s} \mathbf{P}(0) \cdot \mathbf{V}_{m s} .
$$

But $\mathbf{P}(0)$ is a vector in site-space with unity assigned to the initial site of the vacancy, and zeros elsewhere. If this initial site is labeled the $k$ th site in the $p$ th subshell, then $\mathbf{P}(0)$ is orthogonal to all of the basis vectors except the $\mathbf{V}_{p s}$, and

$$
f_{q s}=a_{p q s}\left(V_{p s}\right)_{k}
$$

where $\left(V_{p s}\right)_{k}$ is the $k$ th component of the basis vector $\mathbf{V}_{p s}$. Combining (4), (5), and (6) the probability vector $\mathbf{P}(t)$ may be written

$$
\mathbf{P}(t)=\sum_{q} \sum_{s} \sum_{m} a_{p q s} a_{m q s}\left(V_{p s}\right)_{k} \mathbf{V}_{m s} \exp \left(-\lambda_{q s t}\right) .
$$

To recapitulate the subscripts, $s$ specifies the irreducible representation, or specifies one of the dimensions of a multidimensional irreducible representation, and $m$ both denotes a basis vector within the set belonging to the sth irreducible representation and also identifies a subshell of lattice sites. Together, these subscripts cover the whole dimensionality of the site space. The subscript $q$ indicates one of the eigenvectors belonging to the sth representation-it runs parallel with but is independent of $m$. The initial position of the vacancy is indicated by $p$ (one of the set of subshells identified by the running index $m$ ) and $k$, which gives the site within the subshell.

The vector $\tau_{n}^{\prime}$. The vectors $\tau_{n}^{\prime}$ may also be expressed in terms of the same basis. Each set of basis vectors reflects the symmetry associated with the corresponding irreducible representation. In particular, there will always exist 3 sets, reflecting $x$, $y$, and $z$ symmetry in real space, although they may not necessarily all correspond to the same irreducible representation as they do for cubic crystals. Any function with vector symmetry in real space will, when evaluated at each lattice site, generate a vector in site space that can be expressed as a linear combination of the basis vectors drawn only from the $x$, $y$, and $z$ sets. For these vectors the subscript $s$ will be replaced by $x, y$, or $z$.

Using column vectors in site space, the $x, y$, and $z$ basis vectors for the $n$th subshell may be written

$$
\mathbf{V}_{n x}=L_{n x}^{-1}\left[\begin{array}{c}
x_{n 1} \\
x_{n 2} \\
x_{n 3} \\
\vdots
\end{array}\right] \text {, etc. }
$$

where the $x_{n k}, y_{n k}$, and $z_{n k}$ are the coordinates in real space of the $k$ th site in the $n$th subshell, and the $L_{n x}$, etc., are the normalization constants $\left(\sum_{k} x_{n k}^{2}\right)^{1 / 2}$, etc. Now the vectors $\tau_{n}^{\prime}$ in site space are generated by the function $\cos \theta_{\alpha k}$ evaluated at the lattice sites of the $n$th subshell in real space. This function can also be written

$$
\cos \theta_{\alpha k}=-\frac{\mathbf{r}_{0} \cdot \mathbf{r}_{k}}{\left|\mathbf{r}_{0}\right|\left|\mathbf{r}_{k}\right|}
$$

where $\mathbf{r}_{0}$ is the vector in real space with coordinates $x_{0}, y_{0}$, and $z_{0}$ from the origin to the site from which the tracer made the $\alpha$ th step (the initial site of the vacancy at $t=0$ ), and $\mathbf{r}_{k}$ is the vector to the $k$ th site. Then the vector $\tau_{n}^{\prime}$, in site space, can be written

$$
\boldsymbol{\tau}_{n}^{\prime}=-\frac{1}{\left|r_{0}\right|\left|r_{n}\right|}\left\{x_{0} L_{n x} \mathbf{V}_{n x}+y_{0} L_{n y} \mathbf{V}_{n y}+z_{0} L_{n z} \mathbf{V}_{n z}\right\} .
$$

$\overline{\operatorname{Cos} \theta_{\alpha}}$. With eq (7) for $\mathbf{P}(t)$ and eq (8) for $\boldsymbol{\tau}_{n}^{\prime}$, the scalar product of these two vectors can be written

$$
\begin{aligned}
& \tau_{n}^{\prime} \cdot \mathbf{P}(t)=- \frac{1}{\left|r_{0}\right|\left|r_{n}\right|}\left\{\frac{x_{0}^{2} L_{n x}}{L_{p x}} \sum_{q} a_{p q x} a_{n q x} \exp \left(-\lambda_{n x} t\right)\right. \\
&+\frac{y_{0}^{2} L_{n y}}{L_{p y}} \sum_{q} a_{p q y} a_{n q y} \exp \left(-\lambda_{n y} t\right) \\
&\left.\quad+\frac{z_{0}^{2} L_{n z}}{L_{p z}} \sum_{q} a_{p q z} a_{n q z} \exp \left(-\lambda_{n z} t\right)\right\}
\end{aligned}
$$

where use has been made of the mutual orthogonality of the basis vectors $\mathbf{V}_{m s}$. When this is inserted into eq (1c) for $\overline{\cos \theta_{\alpha}}$ and the integration performed, 
the result is

$$
\begin{aligned}
\overline{\cos \theta_{\alpha}}=\sum_{n} & \frac{w_{n 0}}{\left|r_{0}\right|\left|r_{n}\right|}\left\{\frac{x_{0}^{2} L_{n x}}{L_{p x}} \sum_{q} \frac{a_{p q x} a_{n q x}}{\lambda_{q x}}\right. \\
& \left.+\frac{y_{0}^{2} L_{n y}}{L_{p y}} \sum_{q} \frac{a_{p q y} a_{n q y}}{\lambda_{q y}}+\frac{z_{0}^{2} L_{n z}}{L_{p z}} \sum_{q} \frac{a_{p q z} a_{n q z}}{\lambda_{q z}}\right\} .
\end{aligned}
$$

This equation is in a form that is quite suitable for machine computation. To the irreducible representations denoted by $x, y$, and $z$, there correspond secular equations which can be derived by standard methods. The $q$ th solution to these secular equations provides values for $\lambda_{q x}$, etc. Each solution also gives a set of coefficients, $a_{n q x}$, etc., which are the contributions from the basis vectors $\mathbf{V}_{n x}$ to the $q$ th eigenvector $\mathbf{u}_{q x}$. The vacancy is initially in the $p$ th subshell with coordinates $x_{0}, y_{0}$, and $z_{0}$ in real space. The summation denoted by $n$ is taken over all subshells from which the vacancy can make direct exchanges with the tracer, and by $q$ over all solutions to each secular equation.

Equation (9) may be used to obtain the quantities appearing in Mullen's [5] correlation functions for diffusion in anisotropic lattices. Mullen showed that the important quantities are of the form

$$
\begin{aligned}
& \epsilon_{1}^{\alpha}(A, A)=\frac{\overline{x_{\alpha}^{A} x_{\alpha+1}^{A}}}{\left(x_{\alpha}^{A}\right)^{2}} \\
& \epsilon_{1}^{\alpha}(A, B)=\frac{\overline{x_{\alpha}^{A} x_{\alpha}^{B}}}{\left(x_{\alpha}^{A}\right)^{2}}
\end{aligned}
$$

where $x_{\alpha}^{A}$ is the projection of the $\alpha$ th tracer step on the $x$-principal diffusion axis, and the superscript $A$ indicates that this step is of the $A$ type. The distinction between the $A, B$, etc., types of steps is the same as the distinction among the subshells denoted in eq (9) by the index $n$. From eq (9) it follows that

$$
\epsilon_{1}^{\alpha}(A, B)=\frac{w_{B 0}}{r_{A} r_{B}} \frac{\left(x^{A}\right)^{2} L_{B x}}{L_{A x}} \sum_{q} \frac{a_{A q x} a_{B q x}}{\lambda_{q x}}
$$

where $A, B$ as subscripts denote the subshells corresponding to the $A, B$ types of step.

Boundary conditions. In performing the computations, it is necessary to restrict the vacancy to a finite number of sites around the tracer. This imposes a boundary and necessitates a choice of boundary conditions. A convenient choice is to take a symmetrical boundary by including all sites out to a certain number of steps from the origin. Possible jumps of the vacancy to sites beyond the boundary can then be treated in one of two simple ways. If these jumps are allowed, but the return jumps are not, then the boundary is "transparent" and acts as a perfect sink. This is the boundary condition used by Compaan and Haven [3] and by Mullen [5]. Use of the transparent boundary neglects reflection of the vacancy from the region beyond the boundary. Since the path of the reflected vacancy would, to some extent, be correlated with the initial step, the transparent boundary condition underestimates the value of $\overline{\cos \theta_{\alpha}}$. On the other hand, the jumps through the boundary can be omitted altogether from the matrix $C$, in which case the boundary is totally "reflecting" and the result overestimates $\overline{\cos \theta_{\alpha}}$. If the computations are performed for both conditions, then an upper and a lower limit are obtained for $\overline{\cos \theta_{\alpha}}$.

\section{Self-Diffusion in the FCC Lattice}

The method can be illustrated by calculating $\overline{\cos \theta_{\alpha}}$ for self-diffusion on a fcc lattice. Compaan and Haven [3], using a somewhat different technique to solve essentially the same equations, have given a value for $\overline{\cos \theta_{\alpha}}$ of -0.12268 for this case, to which the results of the present method may be compared.

For the cubic lattices, the $x, y$, and $z$ irreducible representations form a degenerate set, with the same secular equations. For illustrative purposes, we may consider the particular case of the fec lattice, and restrict direct exchanges to the nearest neighbor shell only, without subshells. Then $p=n=1, \quad r_{0}=r_{n}$ $=\left(x_{0}^{2}+y_{0}^{2}+z_{0}^{2}\right)^{1 / 2}$, and eq (9) reduces to the very simple form

$$
\overline{\cos \theta_{\alpha}}=w_{10} \sum_{q} \frac{a_{1 q}^{2}}{\lambda_{q}} .
$$

Furthermore, a simple prescription can be given for the secular equation for the $x, y, z$ irreducible representation ( $T_{1 u}$ in the notation of Eyring, Walter, and Kimball [12]) in the fec lattice. This equation is

$$
C^{\prime} \mathbf{u}_{q}=\lambda_{q} \mathbf{u}_{q}
$$

where $C^{\prime}$ is a matrix whose entries are:

$c_{i i}^{\prime}=$ sum of the jump frequencies of all possible jumps of the vacancy out of a site included in the $i$ th basis vector, into sites not included in the same vector.

$c_{i j}^{\prime}=-g_{i j}\left(\dot{d}_{j} / d_{i j}\right)^{1 / 2} w_{i j}$ where $g_{i j}$ is the number of sites included in the $i$ th basis vector from which jumps into any site included in the $j$ th basis vector are made; $d_{j}$ and $d_{i}$ are the number of nonzero entries in the $j$ th and $i$ th basis vectors, respectively; and $w_{i j}$ is the jump frequency for jumps of the vacancy from a site in the $i$ th basis vector to one in the $j$ th basis vector.

In performing the calculation, the boundary is chosen, thus limiting the number of lattice sites considered explicitly and therefore the dimension of the matrix $C^{\prime}$. The prescription given above is used to write down the matrix, and its eigenvalues and eigenvectors found by numerical computation. The eigenvalues $\lambda_{q}$ and the coefficients $a_{1 q}$ of the first basis vector (corresponding to the sites that are nearest neighbors to the tracer) in the eigenvectors are inserted into eq (10) to give $\overline{\cos \theta_{\alpha}}$.

There is a convergence problem in these calculations, arising from the presence of a boundary of 
finite radius. For self-diffusion, there is no interaction between the tracer and the vacancy to bind them together, so that the vacancy may be expected to make long excursions away from the tracer, and the presence of the boundary may influence the computed value of $\overline{\cos \theta_{\alpha}}$ even for a boundary quite far from the origin. If the boundary be made spherical, an estimate of the rate of convergence of the calculated value of $\overline{\cos \theta_{\alpha}}$ as the radius $R$ of the boundary increases may be made. For this estimate, use is made of the analogy pointed out by Ham [13] between the solutions to a steady-state diffusion problem and the solutions to an electrostatic problem of similar geometry. In the form given by Compaan and Haven [13] eq (2) can be integrated with respect to time from 0 to $\infty$ and written

$$
\begin{aligned}
\int_{0}^{\infty} \frac{d P_{p}}{d t} d t & =1=w \sum_{i}\left(V_{i}-V_{p}\right) \\
\int_{0}^{\infty} \frac{d P_{j}}{d t} d t=0 & =w \sum_{i}\left(V_{i}-V_{j}\right), \quad j \neq p
\end{aligned}
$$

where $w$ is the single jump frequency needed in the self-diffusion case, the subscript $p$ denotes the initial position of the vacancy, and

$$
V_{i}=\int_{0}^{\infty} P_{i}(t) d t
$$

Compaan and Haven noted that eqs (11) are identical to the equations governing current flow in a resistor network. However, these equations apply equally to the charge and potential distribution in a capacitor network. Let the lattice sites be connected by capacitors of capacitance $w$, and let the potential at the $i$ th site be $V_{i}$. Then if a unit charge is placed on the $p$ th site, and zero charge on all others, the potentials on the lattice sites will conform to eqs (11). For the fec lattice, as shown by Compaan and Haven, $\overline{\cos \theta_{\alpha}}$ is given by

$$
\overline{\cos \theta_{\alpha}}=-w\left(V_{1}+2 V_{2}\right)
$$

where $V_{1}$ and $V_{2}$ are the potentials at the sites marked 1 and 2 in figure 1 , when a positive unit charge is at site $p$ and a negative one at site 1 .

An estimate of these potentials may be made when the radius of the boundary is large enough to allow replacing the discrete network by a homogeneous dielectric sphere, with a radius $R$ and with a charge $-q$ placed at $\sqrt{2 a}$ and another one $q$ placed at $-\sqrt{2 a}$, where $a$ is the cation-anion separation (fig. 2). The origin is fixed at zero potential, and the boundary also taken as fixed at zero potential to reproduce the transparent boundary condition described above. The problem is then that of the potential due to fixed charges within a conducting spherical shell held at zero potential. As shown by Abraham and Becker [14], the same potential would be produced by image charges $\pm Q= \pm q R / \sqrt{2 a}$, located at dis-

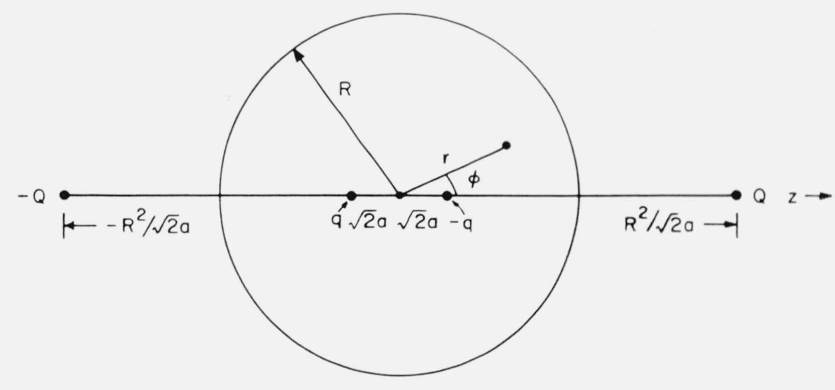

Figure 2. Electrostatic model of the transparent boundary.

$R$ is the radius of the boundary; $r$ and $\phi$ define the position of an arbitrary point within the boundary. Real charges $-q$ and $q$ are placed at the initial vacancy site and the inversion-related position, a distance $\sqrt{2 a}$ from the origin. Image charges $Q$ and $-Q$ are placed on the $Z$ axis $\pm R^{2} / \sqrt{2 a}$ from the origin.

tances $\pm R^{2} / \sqrt{2 a}$ from the origin as shown in figure 2 . If $R \gg a$, then the potential at an arbitrary point within the sphere specified by spherical coordinates $r$ and $\phi$ is

$V=V_{0}+\frac{q}{R}\left\{\frac{4 a^{2}}{R^{2}} \cos \phi+\frac{8 a^{6}}{R^{6}}\left(5 \cos ^{3} \phi-3 \cos \phi\right) \ldots\right\}$

in which the first term $V_{0}$ is due to the real charges within the sphere, and the second term is due to the image charges, and therefore represents the contribution of the boundary.

When potentials at sites 1 and 2 are evaluated using eq (13) and inserted in eq (12), the result is

$\overline{\cos \theta_{\alpha}}=\overline{\cos \theta_{\alpha 0}}+$ constant $\times\left[\left(\frac{a}{R}\right)^{3}-\frac{1}{4}\left(\frac{a}{R}\right)^{7} \ldots\right]$.

This equation may be used as an extrapolation formula to obtain a value for $\overline{\cos \theta_{\alpha 0}}$ from the values of $\overline{\cos \theta_{\alpha}}$ computed for finite boundaries with eq (10). It suggests that the computed value, for large enough values of the radius $R$ of the boundary sphere, should approach the true value as $1 / R^{3}$. Values of $\overline{\cos \theta_{\alpha}}$ computed for both reflecting and transparent boundary, are plotted in figure 3 against $(R / a)^{-3}$, where $R$ has been identified with the distance from the origin to the closest sites from which return jumps were not allowed (transparent boundary) or to which forward jumps were not allowed (reflecting: boundary). The values for the transparent boundary show the expected $R^{-3}$ behavior for large $R$. If eq (14) is used as an extrapolation formula, with arbitrary coefficients, the value of -0.12266 is obtained for $\overline{\cos \theta_{\alpha 0}}$, in essential agreement with the value computed by Compaan and Haven [3].

This $R^{-3}$ convergence is independent of the specific lattice considered, and arises simply from the fact that the tracer position after the $\alpha$ th step is taken as 


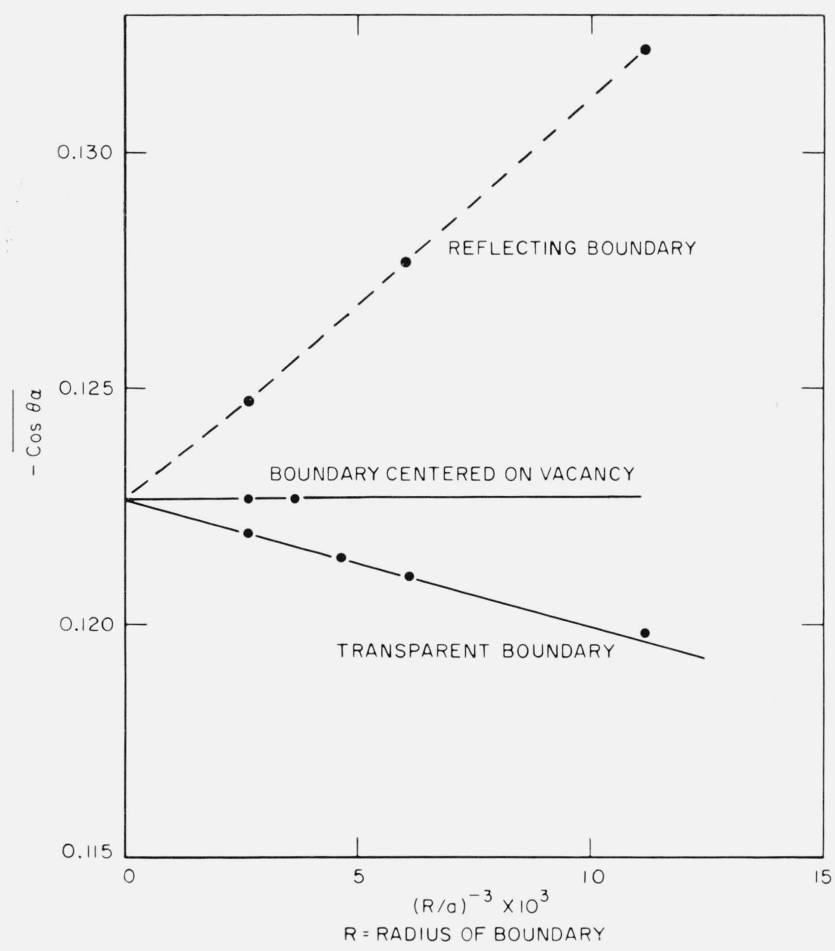

Figure 3. Dependence of $\overline{\cos \theta_{\alpha}}$ upon $(\mathrm{R} / \mathrm{a})^{-3}$, for both transparent and reflecting boundaries centered on the tracer, and for a transparent boundary centered on the vacancy.

The solid line for the transparent boundary was drawn using eq (14) as an extrapolation formula, plotting only the term in $R^{-3}$.

the origin, around which a symmetrical boundary is placed. For impurity diffusion in which the impurity ion and the vacancy are bound together, the excursions of the vacancy out to the boundary will be less likely than in the case of self-diffusion, with no binding. Hence the convergence for impurity diffusion should be more rapid than given by $R^{-3}$.

It is possible to achieve more rapid convergence in the special case of self-diffusion by placing the position of the vacancy after the $\alpha$ th step at the origin, as done by Compaan and Haven [3] and by Mullen [5], although this cannot be done for impurity diffusion. For self-diffusion the tracer is exactly like all other atoms or ions in the crystal. Compaan and Haven showed that for crystals in which the tracer site is a center of symmetry or has a mirror plane, a two-fold, or a three-fold axis of rotation perpendicular to the direction of the $\alpha$ th step, the trajectories of the vacancy that cross the tracer need not be terminated at the tracer. In the electrostatic model, this is equivalent to removing the condition that the tracer site be held at zero potential, and in the language of the relaxation mode analysis, it is equivalent to including jumps of the vacancy out of, as well as into, the tracer site, so that the tracer no longer is a sink for the probability. Under these circumstances, the problem has the symmetry of the site-group at the initial position of the vacancy, and this position can be taken as the origin, with the boundary distributed symmetrically about it, rather than about the tracer. In the electrostatic model for the fec lattice, the spherical boundary can be taken about the initial position of the vacancy as a center, and only at this point is there any charge within the sphere. Such a boundary simply adds a constant to the potential everywhere so that $\overline{\cos \theta_{\alpha}}$, which depends only upon differences in potential, is not affected by the presence of the boundary in the continuum approximation. For a real lattice, there will be a dependence of $\overline{\cos \theta_{\alpha}}$ for boundary radii too small to allow a continuum approximation.

The rapid convergence that results when the boundary can be centered on the vacancy can be illustrated by direct calculation. In the relaxation mode analysis of this case, the initial distribution of probability is spherically symmetric, and the only modes excited will be the spherically symmetric ones, the $A_{1 g}$ for the fec lattice. Equation (10) will be replaced by

$$
\overline{\cos \theta_{\alpha}}=w \sum_{q} \frac{a_{1 q}}{\lambda_{q} \sqrt{n_{1}}}\left\{\frac{a_{1 q}}{\sqrt{n_{1}}}+\frac{2 a_{2 q}}{\sqrt{n_{2}}}-\frac{2 a_{4 q}}{\sqrt{n_{4}}}-\frac{a_{5 q}}{\sqrt{n_{5}}}\right\}
$$

where $a_{i q}$ are the coefficients of the $i$ th basis vector in the $q$ th eigenvector of the $A_{1 g}$ irreducible representation, $\lambda_{q}$ are the corresponding eigenvalues, and $n_{i}$ is the number of sites in the $i$ th shell (there are no subshells for the spherically symmetric modes). Using this equation, and setting a transparent boundary at $R / a=\sqrt{42}$ and $R / a=\sqrt{52}$, we obtained -0.12268 for $\overline{\cos \theta_{\alpha}}$ in both cases (fig. 3). The value had converged even for the smaller boundary, and agrees exactly with Compaan and Haven's value.

It should be noted that the boundary used in this calculation was not exactly identical to that used either by Compaan and Haven [3] or by Mullen [5]. In the present calculations, the boundary was taken as spherical. The distance from the origin to any point on the boundary was the same in all directions, but the number of steps required for the vacancy to reach the boundary was not the same. If, as done by Compaan and Haven, and Mullen, the boundary was taken as a certain number of steps from the origin in all directions, the secular equation could have been somewhat smaller in size and the problem somewhat simpler, without affecting the convergence significantly.

The present method of calculating $\overline{\cos \theta_{\alpha}}$, using the relaxation mode analysis, is basically similar to Mullen's [5] method. Equation (4) of the present paper, giving the probability vector $\mathrm{P}(t)$, can be made equivalent to Mullen's eq (10) by an integration over $t$ from zero to infinity and multiplication of each component by the sum of the frequencies of all jumps out of the corresponding site. Mullen treats only the case of self-diffusion, with the boundary centered on the vacancy. Simplification of the equations is brought about by introducing only sites not symmetry-related to each other under the crystallographic point group. This is equivalent to 
the choice of the $A_{1 g}$ mode as described above. The two treatments lead to matrices of the same dimensionality. In Mullen's formulation, it is necessary to invert the matrix; here, the eigenvalues and eigenvectors must be calculated.

For impurity diffusion, the relaxation mode analysis simplifies the equations by expressing the probability vector $\mathbf{P}(t)$ as a sum of eigenvectors of the matrix $C$, each multiplied by its relaxation function. Equation (1b) then selects only those eigenvectors not orthogonal to $\tau^{\prime}$. The same procedure could be applied to Mullen's eq (10). The initial probability distribution, $\tau(0)$, would be expressed as a sum, each term of which would be equivalent to an eigenvector of Mullen's $T$ matrix. 'The choice of the eigenvectors as a basis would reduce $T$ to its irreducible representations, of which only the vector representation would have to be kept, thus achieving the same reduction in the size of the matrix to be handled as obtained in the relaxation mode analysis. In addition, the boundary would have to be centered on the site of the impurity, since this is the only center of symmetry in the presence of the impurity.

\section{Summary}

Correlation between successive steps of an impurity or tracer as it diffuses through a crystal by the vacancy mechanism can often be expressed in terms of $\overline{\cos \theta_{\alpha}}$, the average of the value of the cosine of the angle between the $\alpha$ th and $(\alpha+1)$ th steps. $\overline{\cos \theta_{\alpha}}$ can be computed for any lattice using the methods of relaxation mode analysis, resulting in eq (9) of this paper, and reducing the problem to a form well suited to machine computation. A symmetrical boundary is taken about the impurity or tracer. When the boundary is transparent, so that the vacancy does not return once it has penetrated the boundary, the computation gives a lower limit to the magnitude of $\overline{\cos \theta_{\alpha}}$. An upper limit is produced when the boundary is totally reflecting. The computed values of $\overline{\cos \theta_{\alpha}}$ for self-diffusion with the transparent boundary converge as $1 / R^{3}$, where $R$ is the distance from the origin to sites just beyond the boundary. For impurity diffusion in which the vacancy and impurity are attracted to each other, the convergence should be somewhat more rapid.
The method has been illustrated by computing $\overline{\cos \theta_{\alpha}}$ for self-diffusion in the fcc lattice. The $1 / R^{3}$ convergence was demonstrated, and an extrapolated value of 0.12266 obtained, in essential agreement with the value of 0.12268 given by Compaan and Haven [3]. For the special case of self-diffusion, Compaan and Haven showed that for sufficiently symmetric crystals, the origin can be placed at the initial position of the vacancy and a symmetrical boundary placed around it, producing much more rapid convergence. Computations for this model were also made for the fcc lattice, with two sizes of boundary, and both gave values in exact agreement with Compaan and Haven's value.

The author is indebted to A. B. Lidiard for the original suggestion that the relaxation mode analysis could be used to calculate $\overline{\cos \theta_{\alpha}}$, and to R. C. F. McLatchie for performing the computations.

\section{References}

[1] J. Bardeen and C. Herring in Imperfections in Nearly Perfect Crystals p. 261 (John Wiley \& Sons, New York, 1952).

[2] A. D. LeClaire and A. B. Lidiard, Phil. Mag 1, 518 (1956).

[3] K. Compaan and Y. Haven, Trans. Far. Soc. 52, 786 (1956); 54, 1498 (1958).

[4] J. R. Manning, Phys. Rev. 116, 819 (1959); 128, 2169 (1962).

[5] J. G. Mullen, Phys. Rev. 124, 1723 (1961).

[6] H. B. Huntington and P. B. Ghate, Phys. Rev. Letters 8, 421 (1962).

[7] Y. Haven and J. H. Van Santen, J. Chem. Phys. 22, 1146 (1954); Nuovo Cimento '\%, 605 (1958).

[8] J. B. Wachtman, Jr., Phys. Rev. 131, 517 (1963)

[9] A. D. Franklin, J. Res. NBS 6ra, (Phys. and Chem.) No. 4, 291 (1963).

[10] S. Bhagavantam and P. V. Pantulu, Proc. Indian Acad. Sci. 58, 183 (1963).

[11] A. D. Franklin, A. Shorb, and J. B. Wachtman, Jr., J. Res. NBS 68A, (Phys. and Chem.) No. 5, 425 (1964).

[12] H. Eyring, J. Walter, and G. E. Kimball, Quantum Chemistry (John Wiley \& Sons, New York, 1944).

[13] F. S. Ham, J. Phys. Chem. Solids 6, 335 (1958).

[14] M. Abraham and R. Becker, The Classical Theory of Electricity and Magnetism, p. 68, translated into English by J. Dougall (Blackie and Son, Ltd., Glasgow, 1937).

(Paper 69A4-350) 